\title{
Deep sequencing of pre-translational mRNPs reveals hidden flux through evolutionarily conserved alternative splicing nonsense-mediated decay pathways
}

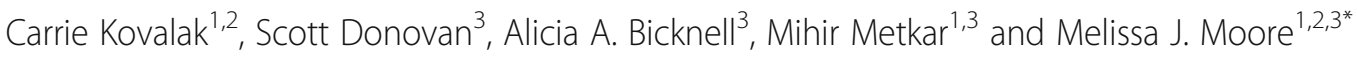

\author{
* Correspondence: melissa.moore@ \\ umassmed.edu \\ 'RNA Therapeutics Institute, \\ University of Massachusetts Medical \\ School, Worcester, MA 01605, USA \\ ${ }^{2}$ Department of Biochemistry and \\ Molecular Pharmacology, University \\ of Massachusetts Medical School, \\ Worcester, MA 01605, USA \\ Full list of author information is \\ available at the end of the article
}

\begin{abstract}
Background: Alternative splicing, which generates multiple mRNA isoforms from single genes, is crucial for the regulation of eukaryotic gene expression. The flux through competing splicing pathways cannot be determined by traditional RNA-Seq, however, because different mRNA isoforms can have widely differing decay rates. Indeed, some mRNA isoforms with extremely short half-lives, such as those subject to translation-dependent nonsense-mediated decay (AS-NMD), may be completely overlooked in even the most extensive RNA-Seq analyses.
\end{abstract}

Results: RNA immunoprecipitation in tandem (RIPiT) of exon junction complex components allows for purification of post-splicing mRNA-protein particles (mRNPs) not yet subject to translation (pre-translational mRNPs) and, therefore, translationdependent mRNA decay. Here we compare exon junction complex RIPiT-Seq to whole cell RNA-Seq data from HEK293 cells. Consistent with expectation, the flux through known AS-NMD pathways is substantially higher than that captured by RNASeq. Our RIPiT-Seq also definitively demonstrates that the splicing machinery itself has no ability to detect reading frame. We identify thousands of previously unannotated splicing events; while many can be attributed to splicing noise, others are evolutionarily conserved events that produce new AS-NMD isoforms likely involved in maintenance of protein homeostasis. Several of these occur in genes whose overexpression has been linked to poor cancer prognosis.

Conclusions: Deep sequencing of RNAs in post-splicing, pre-translational mRNPs provides a means to identify and quantify splicing events without the confounding influence of differential mRNA decay. For many known AS-NMD targets, the nonsense-mediated decay-linked alternative splicing pathway predominates. Exon junction complex RIPiT-Seq also revealed numerous conserved but previously unannotated AS-NMD events.

Keywords: Exon junctions, mRNA isoforms, AS-NMD, RIPiT-Seq, Pre-translational mRNPs, Splicing noise 


\section{Background}

A central mechanism underlying metazoan gene expression is alternative pre-mRNA processing, which regulates the repertoire of mRNA isoforms expressed in various tissues and under different cellular conditions. Extensive deep sequencing of RNA (RNASeq) has revealed that $\sim 95 \%$ of human protein-coding genes are subject to alternative splicing (AS) [1, 2], with current estimates suggesting $\sim 82,000$ different protein-coding mRNA isoforms generated from $\sim 20,000$ protein-coding genes [3]. Thus, production of alternative mRNA isoforms massively expands the protein repertoire that can be expressed from a much smaller number of genes $[4,5]$. But cells also need to control how much of each protein is made. Although transcriptional control is often considered the predominant mechanism for modulating protein abundance, emerging evidence indicates that post-transcriptional regulatory mechanisms are crucial as well.

Not all mRNA variants are protein-coding. Nearly 15,000 human mRNAs in the Ensembl database (release 93) are annotated as nonsense-mediated decay (NMD) targets [3]. NMD is a translation-dependent pathway that both eliminates aberrant mRNAs with malformed coding regions (i.e., those containing premature termination codons due to mutation or missplicing) and serves as a key mechanism for maintenance of protein homeostasis [6]. This protein homeostasis function is mediated by AS linked to NMD (AS-NMD), wherein the flux through alternate splicing pathways that result in proteincoding and NMD isoforms is subject to tight control [7]. These NMD isoforms harbor a premature termination codon either due to frameshifting or inclusion of a poison cassette exon. Because NMD isoforms are rapidly eliminated after the first or "pioneer" round of translation, only protein-coding isoforms result in appreciable protein production (Fig. 1a, bottom). Thus increasing or decreasing flux through the NMD splicing pathway decreases or increases protein production, respectively. Although AS-NMD was originally described as a mechanism by which RNA-binding proteins (e.g., SR and hnRNP proteins) could autoregulate their own synthesis, recent work indicates that AS-NMD is much more pervasive, tuning abundance of many other proteins such as those involved in chromatin modification and cellular differentiation [8].

The true extent to which AS-NMD contributes to protein homeostasis can only be appreciated by determining flux through the splicing pathways that alternately produce protein-coding and NMD isoforms. Transcriptome-wide assessment of mRNA isoform abundance generally relies on RNA-Seq, which provides a static snapshot of the species present in the sample at the time of collection. Because NMD isoforms are so rapidly decayed, they are generally underrepresented in RNA-Seq datasets. Thus, a single RNA-Seq snapshot is generally uninformative as to synthetic flux through proteincoding and NMD splicing pathways.

An alternate means to assess protein-coding and NMD pathway flux is to capture newly synthesized mRNAs after splicing completion but prior to translation. Late in the splicing cycle, the exon junction complex (EJC) is deposited upstream of at least $80 \%$ of exon-exon junctions (canonical; cEJCs) and at multiple other sites throughout the length of spliced exons (noncanonical; ncEJCs) $[9,10]$. Upon nucleocytoplasmic export, the pioneer round of translation removes EJCs within the 5' UTR and CDS regions, with EJCs remaining downstream of stop codons being key mediators of NMD [11]. Pre-translational mRNPs can be selectively isolated by tandem immunoprecipitation of epitope-tagged and untagged EJC 


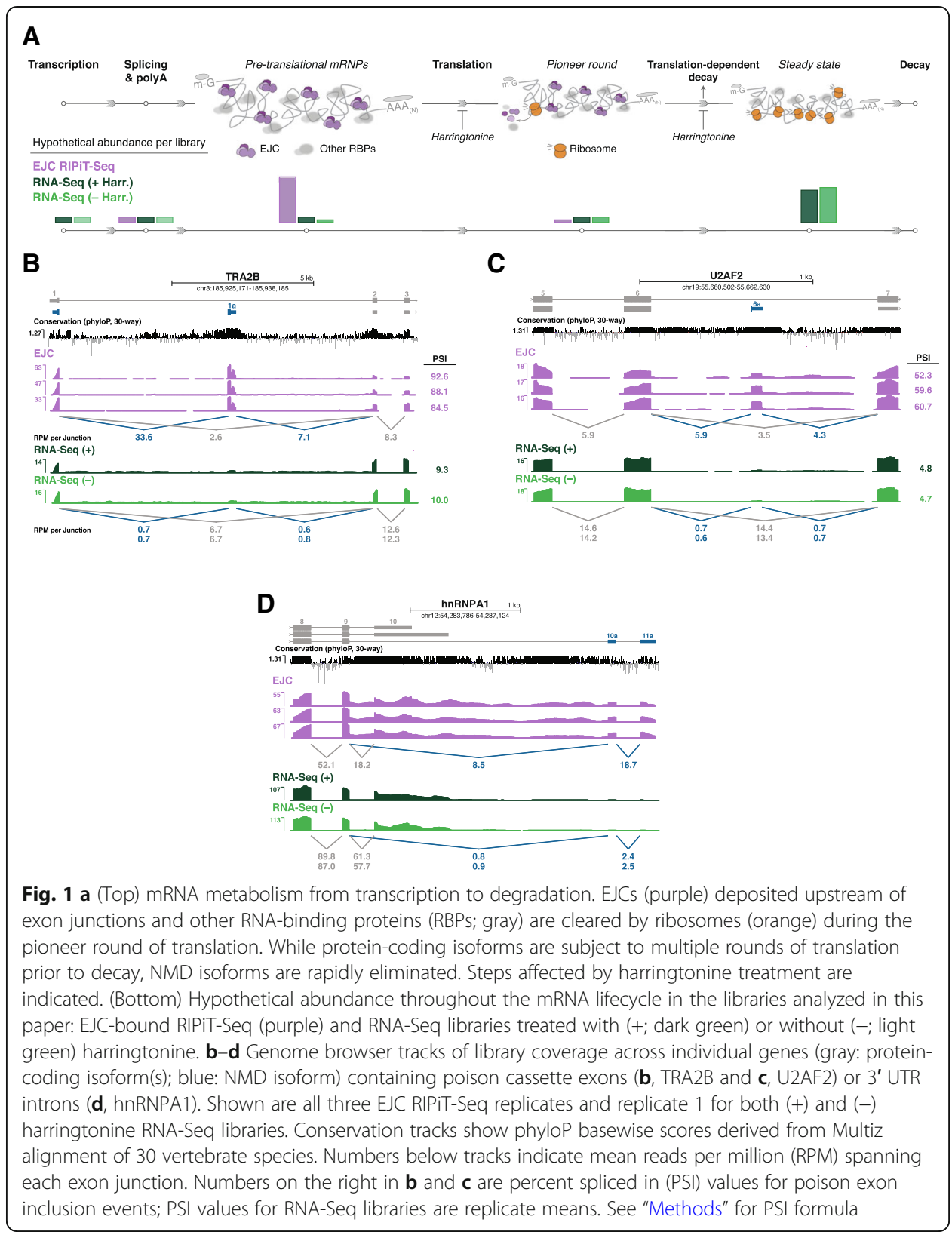

components, a technique known as RNA:protein immunoprecipitation in tandem (RIPiT) [12]. Deep sequencing library preparation from RIPiT samples (RIPiTSeq) has previously enabled us to map the positions of canonical and noncanonical EJCs on spliced transcripts [9] and to investigate the RNA packing principles within pre-translational mRNPs [13].

Here, we compare libraries from pre-translational mRNPs (EJC RIPiT) isolated from subconfluent HEK293 cells to matched RNA-Seq libraries (Fig. 1a). As expected, EJC RIPiT-Seq libraries are enriched for transcript isoforms destined for translationdependent decay. By providing a window into the repertoire of transcripts generated by splicing but prior to translation-dependent decay, EJC RIPiT-Seq libraries provide a more accurate record of the flux through various alternative processing pathways than 
does standard RNA-Seq. Importantly, EJC RIPiT-Seq libraries enabled us to identify numerous new evolutionarily conserved poison cassette exons that had previously eluded annotation.

\section{Results}

\section{EJC and RNA-Seq libraries}

In our recent study investigating the organizing principles of spliced RNPs [13, 14], we generated three biological replicates from subconfluent HEK293 cells of EJC-bound RNAs partially digested with RNase T1 during RNP purification (Fig. 1a). Paired-end deep sequencing of these EJC RIPiT-Seq libraries resulted in 19-25 million mate pairs each (Additional file 1: Table S1). To enable comparison to RNA-Seq for the current study, we created and sequenced rRNA-depleted whole cell RNA-Seq libraries (84-93 million mate pairs each) [15] wherein the captured fragments were of similar length (220-500 nts) to our previously published EJC RIPiT-Seq libraries. The new RNA-Seq libraries were generated from cultures (three biological replicates each) that were $(+)$ or were not $(-)$ subjected to a $1 \mathrm{~h}$ pre-treatment with harringtonine. Harringtonine, a translation initiation inhibitor, is used in the EJC RIPiT-Seq protocol to enrich for pretranslational mRNPs $[9,12,16]$.

For all libraries, raw reads were aligned to the Genome Reference Consortium Human Build 38 (GRCh38.p12) [3] using STAR (v2.5.3a) [17] after first filtering out those mapping to repeat RNAs [18]. To minimize any effects due to misalignment in ensuing analyses, mismatches were limited to three per read, with gaps caused by deletions or insertions being strongly penalized. These strict mapping parameters resulted in 6-10 million and 60-76 million aligned pairs for the EJC RIPiT-Seq and RNA-Seq libraries, respectively (Additional file 1: Table S1). For quantification, we limited all analyses to unique reads with high mapping quality (MAPQ $\geq 5$ ). For all libraries, we used Kallisto (v0.44.0) to derive expression values for the $\sim 200,000$ annotated transcripts in GRCh38.p12 [3]. Examination of per-transcript abundance revealed high concordance ( $\geq 0.93$ to 0.99 ) among biological replicates (Additional file 1: Figure S1A). Therefore, all subsequent quantitative analyses utilized merged biological replicate data.

EJC libraries are enriched for spliced transcripts and translation-dependent decay targets To assess the relative abundance of NMD targets in EJC and RNA-Seq libraries, we first examined read coverage on known AS-NMD genes. The SR proteins TRA2B and U2AF2 negatively regulate their own expression by promoting inclusion of a highly conserved poison cassette exon containing a premature termination codon (Fig. 1b, c). Although these poison exons were detectable in all library types, they were substantially more abundant in the EJC libraries. Whereas the RNA-Seq libraries returned low poison exon inclusion values (percent spliced in; PSI; $9.3-10.0 \%$ and $4.7-4.8 \%$, respectively), the EJC RIPiT-Seq libraries indicate much higher inclusion rates (88.4\% and $57.5 \%$, respectively). Thus, for both TRA2B and U2AF2, the predominant splicing pathway in HEK293 cells under standard growth conditions is poison exon inclusion. Similar trends were observed for other known AS-NMD targets (Additional file 1: Figure S1B-D), including hnRNPA1 where the AS-NMD isoform results from 3' UTR splicing as a 
consequence of alternative polyadenylation (Fig. 1d). Importantly, the (-) and (+) harringtonine RNA-Seq libraries exhibited nearly identical AS-NMD isoform abundances. Thus a 60-min inhibition of translation was insufficient to substantially change AS-NMD isoform abundance in whole cell RNA-Seq libraries from subconfluent HEK293 cells. In contrast, the substantial differences between the EJC RIPiT-Seq and RNA-Seq quantitations for these previously documented ASNMD isoforms clearly illustrate the advantage provided by EJC RIPiT-Seq for more accurately assessing flux through alternative processing pathway resulting in mRNA isoforms with widely different decay rates.

In GRCh38.p12, every transcript isoform is given a specific annotation [19]; relevant annotations in protein-coding genes are "protein-coding," "NMD," "NSD," "retained intron," and "processed transcript," with the latter being a catch-all for transcripts not clearly attributable to any other category. NSD (nonstop decay) is another translation-dependent mRNA degradation pathway that eliminates transcripts having no in-frame stop codon [20]. Retained intron transcripts are generally subject to translation-dependent decay driven by in-frame stop codons in the intronic regions. For transcripts detectable in our libraries [TPM $>0$ in all replicates of a particular library type: EJC and (+) or (-) RNA-Seq], the number of exon junctions (i.e., positions at which introns were removed) ranged from 0 to $>100$ per protein-coding isoform and 1 to 69 per NMD isoform (Fig. 2a). As expected, protein-coding isoforms having no exon junctions were less abundant in the EJC libraries than in RNA-Seq libraries (Fig. 2b, top). In contrast, spliced protein-coding isoforms containing 5 or more exon junctions were enriched in EJC libraries, with the degree of enrichment increasing with exon junction number. For each exon junction number bin (i.e., 1-4, 5-10 and 10+), NMD isoforms were even more enriched in EJC libraries than were protein-coding isoforms (Fig. 2b, bottom). EJC library enrichment was also readily discernible for NMD, NSD, retained intron, and processed transcript isoforms (Fig. 2c and Additional file 1: Figure S2A), with median enrichments falling between 1.8 and 2.6-fold (Additional file 1: Figure S2B). Because the high degree of overlap between alternate transcript isoforms from individual genes confounds individual isoform abundance quantification by algorithms such as Kallisto [21, 22], we performed an additional analysis examining only those exon junctions (or intron-exon boundaries for retained introns) not shared between multiple GRCh38.p12 transcripts. This revealed an even greater enrichment of NMD, NSD, retained intron, and processed transcript isoforms in EJC than RNASeq libraries (Fig. 2d), with median fold enrichments ranging from 2.5 to 2.9-fold (Fig. 2e; note that the number of NSD transcripts with unique exon junctions was insufficient to provide statistical significance). Thus, EJC RIPiT-Seq libraries are highly enriched for spliced transcripts subject to rapid clearance by translationdependent decay.

\section{EJC libraries capture previously unannotated exon junctions}

We next wondered whether EJC libraries might contain transcript isoforms that had heretofore escaped annotation due to their low abundance in RNA-Seq. Such isoforms should contain previously unannotated exon junctions. To identify all annotated exon 


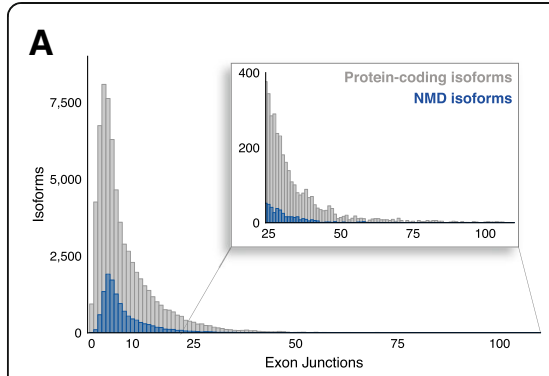

C
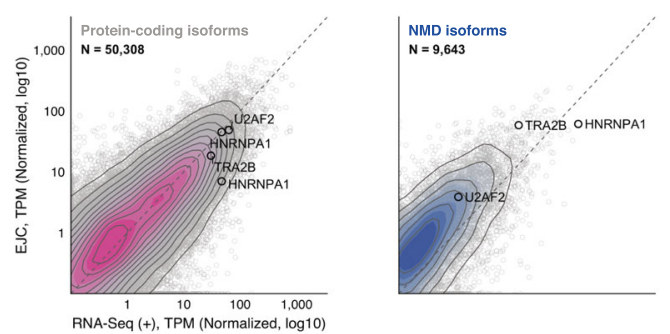

B
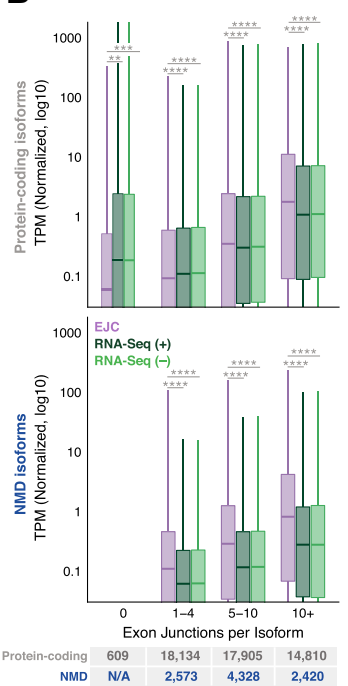

D
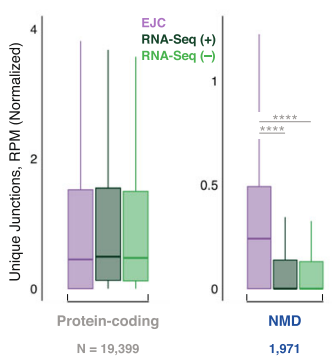

E
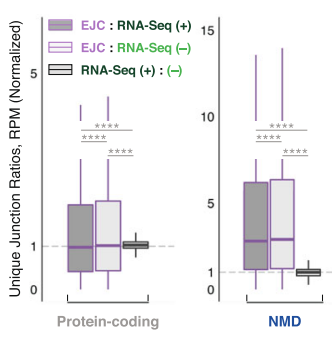

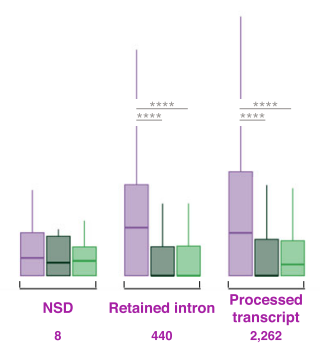

440 262

Fig. 2 a Distribution of the number of exon junctions in all annotated protein-coding (gray) or NMD (blue) transcripts. b Distribution of protein-coding (top) and NMD (bottom) transcripts per million (TPM) in each library type (colors as in Fig. 1a), binned based on indicated number of exon junctions per transcript. Numbers in bottom table: total number of expressed isoforms per exon junction number bin. c Scatterplots comparing TPMs between EJC RIPiT-Seq and RNA-Seq (+) libraries for protein-coding (left) and NMD (right) isoforms. Transcripts from Fig. 1 are noted. N: number of detected transcripts (out of all annotated transcripts of that type). Dashed black line: $x=y$. $\mathbf{d}$ Distribution of read counts at unique junctions, binned based on transcript biotypes [3]. Numbers below: Total number of unique junctions detected in our libraries per transcript biotype. e Ratios of read counts at unique junctions between two indicated library types, binned based on transcript biotype [3]. For $\mathbf{b}$, d, e, results of one-way ANOVA and Tukey's post hoc significance tests comparing EJC RIPiT-Seq to RNA-Seq libraries are indicated as ${ }^{*} P<0.05$, ${ }^{*} P<0.01$, ${ }^{* * *} P<0.005,{ }^{* * * *} P<0.0001$

junctions, we integrated the RefSeq (hg38) [23], Ensembl (GRCh38.p12) [3], GENCODE (v29) [24], and Comprehensive Human Expressed SequenceS (CHESS) transcriptome annotations to create a comprehensive reference file containing 575,837 known introns (Additional file 2: Table S2). While Ensembl and GENCODE are largely identical [25], our analysis revealed 240 junctions in Ensembl GRCh38.p12 that were not in GENCODE v29, and 4554 junctions in GENCODE v29 that were not in Ensembl GRCh38.p12 (Additional file 2: Table S2). CHESS is derived from 9795 RNA-Seq samples from diverse cell types in the GTEx collection, so represents the most complete compendium of human transcripts reported to date [26]. Nonetheless, while CHESS found 118,043 new exon junctions not previously annotated in RefSeq, Ensembl, or GENCODE, 106,223 other junctions present in RefSeq, Ensembl, and/or GENCODE were not returned by the CHESS pipeline (Fig. 3a). This lack of 

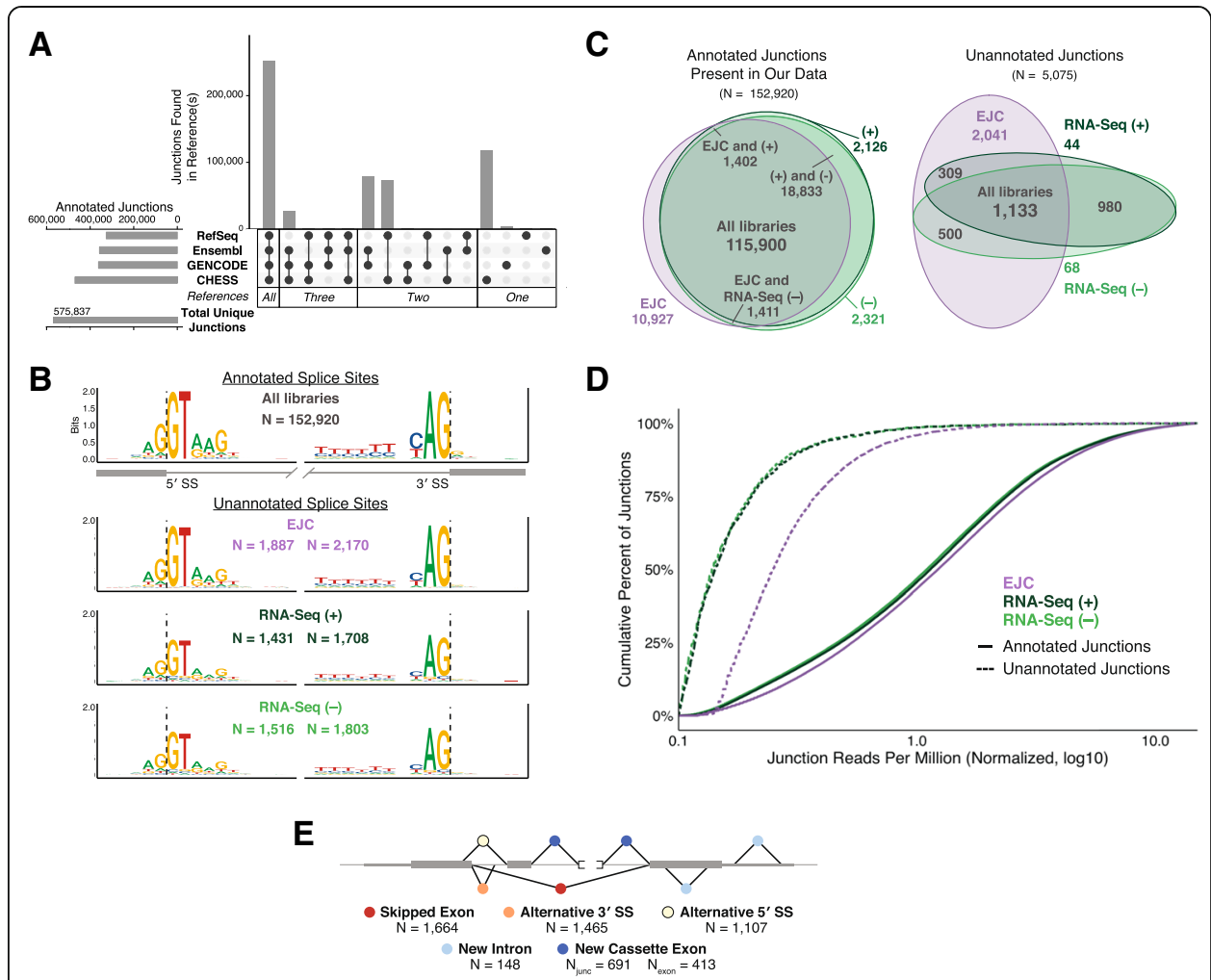

Fig. 3 a Comparison of annotated exon junctions among the transcriptomes sourced from RefSeq (hg38), Ensembl (GRCh38.p12), GENCODE (v29), and CHESS (v2.1). Horizontal bars: total junctions in each reference set; vertical bars: intersections of indicated reference sets. Bar graphs created with UpSetR [27]. b Sequence motifs for 5' (left) and 3' (right) splice sites used in annotated junctions observed in at least one analyzed library type (top) and for previously unannotated splice sites in indicated library type (bottom). Sequence logos were generated in R using ggseqlogo [28]; letter height signifies the relative abundance of that nucleotide at each position. N: number of splice sites contributing to each logo. Note that the number of unannotated junctions (6363) is greater than the total number of unannotated splice sites because many unannotated junctions combine an annotated and unannotated splice site (i.e., alternative 5' or 3' splice sites). c Venn diagram of annotated and previously unannotated junctions (numbers indicated) shared between library types. Venn diagrams made with eulerr [29]. d Cumulative histogram of exon junction reads (RPM) at annotated (solid line) and previously unannotated (dashed line) junctions in each library type (colors as in Fig. 1a). e Schematic of unannotated splicing events separated by event type: skipped exon (red); alternative 3' (orange) or 5' (yellow) splice site; new intron (light blue); new cassette exon (dark blue). $\mathrm{N}$ : number of observed events; for new cassette exons, both the number of observed unannotated junctions and number of new exons are shown

concordance shows that even the most comprehensive RNA-Seq data analyses are unlikely to annotate all bona fide splicing events.

To identify annotated and unannotated exon junctions in our EJC and RNA-Seq libraries, we considered only those reads that cross an exon junction. The position of an exon junction in an individual read can be found by examining the "N operation" in the CIGAR string, which indicates the locations and lengths of gaps inserted during alignment to genomic DNA (Additional file 1: Figure S3). We further required that any candidate junction (1) occurs within an annotated gene; (2) has reads with $\geq 15 \mathrm{nt}$ aligning on both sides of the junction $(\geq 90 \%$ exact sequence match on each side); (3) be detectable in all replicates of a particular library type; and (4) has a mean read count $\geq 2$ per library type (Additional file 1: Figure S3). Using these criteria, we identified 152,920 junctions contained in the RefSeq/ 
Ensembl/GENCODE/CHESS reference file (annotated junctions) and 6363 unannotated junctions. MEME analysis of the latter revealed the $5^{\prime}$ and $3^{\prime}$ splice site consensus motifs for the major spliceosome, although at somewhat lesser strength (bits) than annotated junctions (Fig. 3b). To limit our analysis to events most likely representing real splicing events (as opposed to mapping artifacts), we subsequently only considered the 5075 previously unannotated junctions where the putative intron began and ended with dinucleotides GT-AG or AT-AC to include excision events mediated by both the major and minor spliceosomes (Additional file 3: Table S3).

The majority (76\%) of previously annotated exon junctions meeting our detection criteria in protein-coding genes (Additional file 1: Figure S3) were present in all three library types (Fig. 3c, left). There was less concordance, however, with respect to unannotated junctions, with the EJC libraries having many more unannotated junctions than either $(+)$ or $(-)$ harringtonine RNA-Seq (Fig. 3c, right). Consistent with this, unannotated junctions were supported by more reads per million mapped (RPM) in EJC than in either RNA-Seq library (Fig. 3d; $p=2.2 \mathrm{E}-16$, Kolmogorov-Smirnov test), while read coverages over annotated junctions were remarkably similar between library types. The major class $(51 \%)$ of the new junctions were new alternative $5^{\prime}$ or $3^{\prime}$ splice sites (i.e., that combined a known $3^{\prime}$ or $5^{\prime}$ splice site with a previously unannotated 5' or 3' splice site, respectively) (Fig. 3e). Other categories were previously unannotated exon skipping events (33\%), new cassette exons (14\%), and new introns (3\%).

\section{Relationship of new splicing events to reading frame}

Previous analyses of low abundance, unannotated splicing events in RNA-Seq data have revealed a strong tendency for such events to maintain reading frame [30, 31]. To investigate whether this is due to some inherent ability of the splicing machinery to detect reading frame in the nucleus [32, 33], or simply due to translation-dependent decay of out-of-frame events, we determined the distance from each previously unannotated splice site meeting our selection criteria to the nearest annotated splice site observed in any of our three library types. In all, 250 and 522 unannotated 5' and 3' splice sites, respectively, occurred within 15 nts of an annotated $5^{\prime}$ or $3^{\prime}$ splice site. Comparison of unannotated-to-annotated splice site distance aggregation plots between the three library types revealed both similarities and differences (Fig. 4a). Around annotated 5 ' splice sites, all three libraries displayed similar patterns, with the greatest unannotated usage being at intron position +5 , consistent with the preference for a $\mathrm{G}$ and a $\mathrm{T}$ at positions +5 and +6 , respectively, in the human $5^{\prime}$ splice site consensus sequence (Fig. $3 \mathrm{~b}$ ) and the prevalence of GT dinucleotides at this position in this set of $2505^{\prime}$ splice sites (dotted gray line in Fig. 4a). More notable was the pattern near 3' splice sites, where positions $+3,+4$, and +5 in the downstream exon exhibited the highest unannotated usage. Strikingly, whereas the RNA-Seq libraries were strongly skewed toward position +3 , all three positions were highly represented in the EJC libraries, with their usage more reflective of the number of available AG's at these positions (dotted gray line in Fig. 4a). Comparison of fractional abundance [unannotated read counts/(unannotated + annotated read counts)] at individual sites confirmed that whereas the EJC and RNA-Seq libraries exhibited similar utilization at position +3 , 


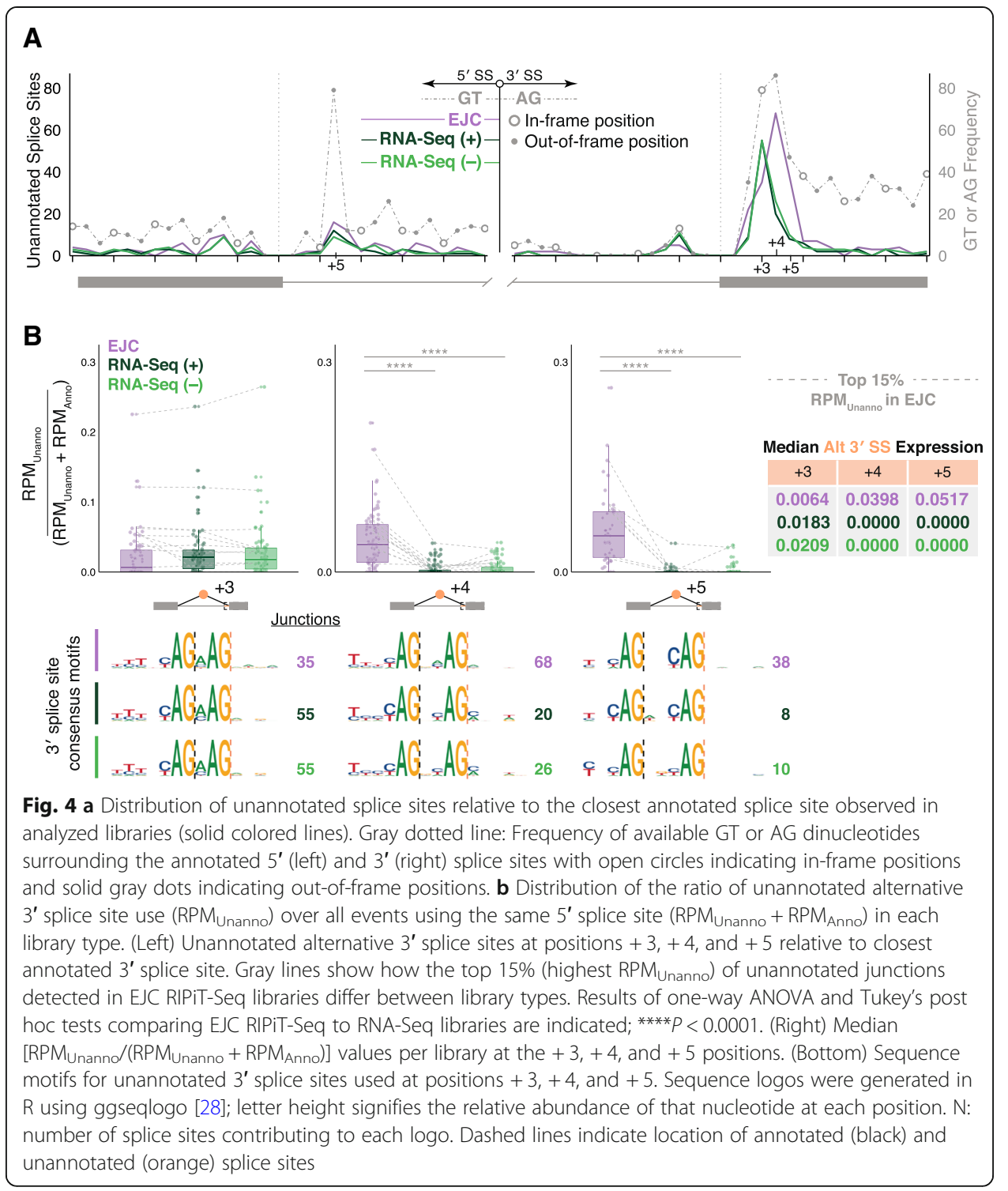

utilization of positions +4 and +5 was much more prominent in the EJC than either RNA-Seq library (Fig. 4b). These observations strongly support a model in which outof-frame splicing events are rapidly eliminated by NMD, resulting in their underrepresentation in RNA-Seq libraries. Because downstream AG utilization in the EJC libraries so closely paralleled their availability, we conclude that (at least with regard to 3 ' splice sites) the splicing machinery has no ability to read frame.

\section{Evolutionary conservation versus splicing noise}

Regardless of reading frame, most unannotated splicing events are likely due to "splicing error" [34] or "splicing noise" [31]. Splicing noise results from spurious utilization of cryptic splice sites that are not evolutionarily conserved. To assess both evolutionary conservation and splice site strength, we calculated mean basewise phyloP 30-way vertebrate conservation [35] and MaxENT (a generally accepted measure of how well a 
particular splice site matches the consensus) [36] scores for both annotated and unannotated splice sites, using the same $5^{\prime}$ and $3^{\prime}$ splice site window sizes (9 and 23 nts, respectively) for both calculations (Fig. 5a). We also calculated conservation and MaxENT scores for sequences chosen at random from inside annotated genes and containing either GT or AG at the appropriate position within the $5^{\prime}$ or $3^{\prime}$ splice site window, respectively. Plotting MaxENT versus conservation revealed markedly different distributions between annotated splice sites and random GT- and AG-containing sequences (Fig. 5b and Additional file 1: Figure S4A-B), with annotated sites being notably skewed toward higher values for both measures. In contrast, whereas unannotated splice sites were similarly distributed as annotated splice sites with regard to MaxENT, the majority exhibited conservation scores more similar to random than annotated splice sites (Fig. 5c and Additional file 1: Figure S4C). For the random sequences, 95\% had $5^{\prime}$ and $3^{\prime}$ splice site conservation scores below 1.04 and 0.63 , respectively. Using these values as cutoffs to filter out the majority of events likely due to splicing noise (although this may be unnecessarily conservative for 3' splice sites due to the high degree of overlap between the annotated and random conservation scores) left us with $453(23 \%)$ and 651 (28\%) evolutionarily conserved unannotated 5' and 3' splice sites,

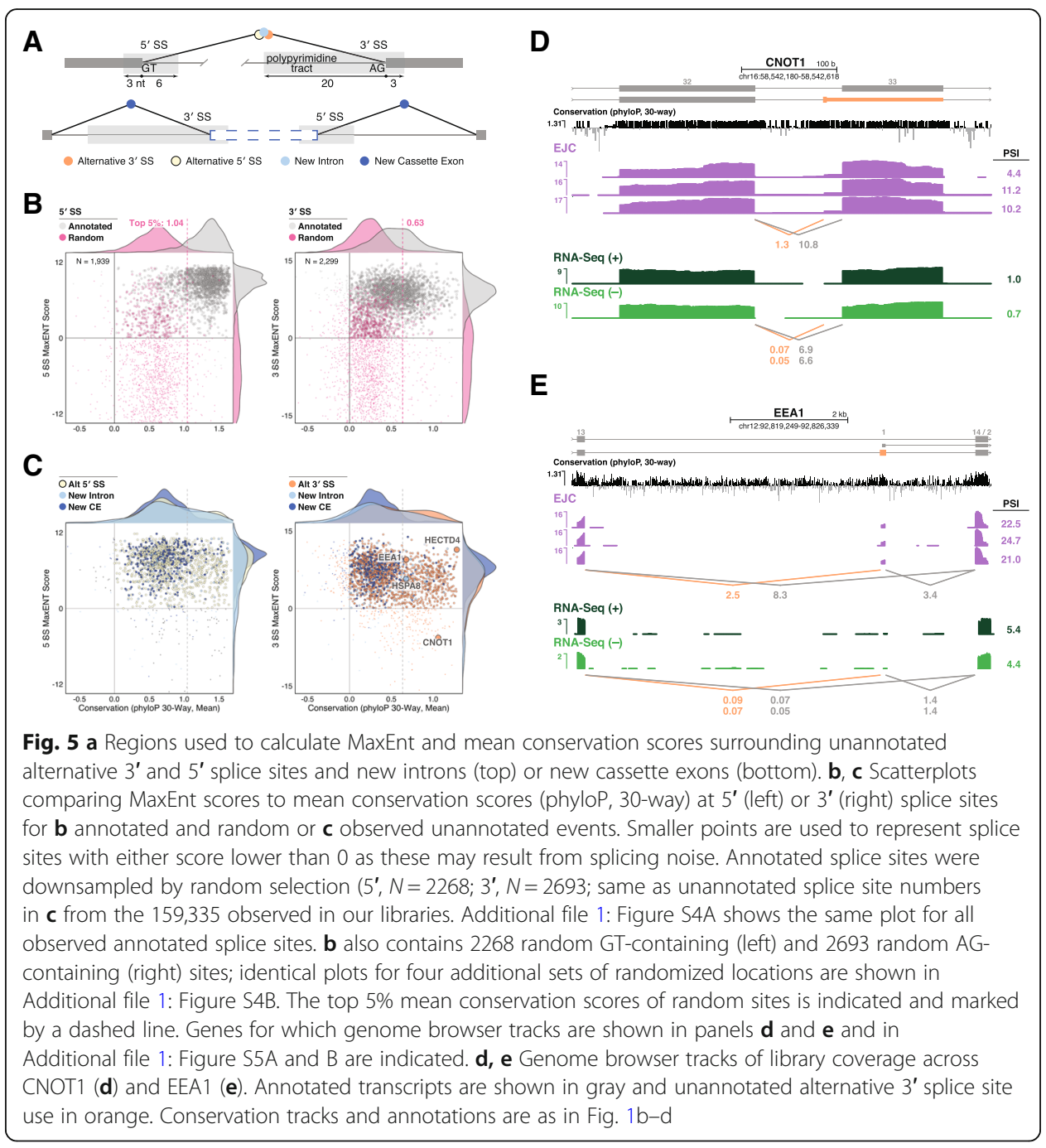


respectively (Additional file 3: Table S3). The majority of these occurred within annotated protein-coding exons, so their conservation is likely driven by amino acid conservation and not as a requirement for recognition by the splicing machinery (see Additional file 1: Figure S5A for an example). Almost all of the new evolutionarily conserved introns (i.e., both the $5^{\prime}$ and $3^{\prime}$ splice sites were previously unannotated, but exhibited high conservation) also fell into this category. For the new introns, calculation of percent intron retention (PIR) in the EJC libraries revealed highly inefficient splicing (mean PIR $=84 \%$ ); individual examination of those exhibiting the highest number of exon junction reads in the EJC libraries led to no findings of particular note. Thus, the new introns likely constitute splicing noise due to low level spliceosome assembly on sites within exons that by happenstance resemble splice site consensus sequences. In contrast, examination of unannotated 3' splice sites occurring within introns uncovered a conserved alternative splicing event in the HECTD4 (HECT domain E3 ubiquitin protein ligase 4) gene that adds 9 amino acids into the middle of the protein (Additional file 1: Figure S5B); this spliced isoform is currently annotated in mouse RefSeq and GENCODE, but not in humans. Other alternative 3' splice sites in the CNOT1 and EEA1 genes generate AS-NMD isoforms (Fig. 5e, f), the latter due to creation of a new poison cassette exon.

\section{New evolutionarily conserved poison cassette exons}

Having found examples of new AS-NMD isoforms generated by unannotated 3' splice sites, we were interested to investigate which of the new cassette exons identified here might also function in this capacity. Of our 413 new cassette exons (Fig. 3e), 383 (93\%) occurred in protein-coding genes; the remainder occurred in pseudogenes and ncRNAs. Based on the data in Fig. 1, poison exons should exhibit higher abundance in EJC than in RNA-Seq libraries. Consistent with this, 318/383 (83\%) were solely detectable in the EJC libraries, with the remainder averaging 26- and 24-fold higher abundance in EJC than in RNA-Seq libraries treated with $(+)$ or without $(-)$ harringtonine, respectively (Fig. 6a). Of the 376 new cassette exons detectable in EJC libraries, $70 \%$ were frameshifting (i.e., not a multiple of 3 nts long). Individual inspection of the 25 most abundant non-frameshifting exons revealed that $80 \%$ contained an in-frame stop codon. Therefore, as expected, the vast majority of our newly identified cassette exons likely function as poison exons.

To assess whether any of the new cassette exons constitute conserved regulatory elements, we calculated mean phyloP 30-way conservation scores across the entire exon. Combining these exon conservation scores (white to dark blue in Fig. 6b) with the previously calculated $5^{\prime}$ and $3^{\prime}$ splice site conservation scores (Fig. 5b) revealed a set of 20 previously unannotated cassette exons exhibiting both high internal (phyloP score $\geq 1$ ) and high splice site ( $\geq 1$ for both splice sites) conservation (Fig. 6b, right; Additional file 3: Table S3). Among these, the most highly represented in our datasets was a new $94 \mathrm{nt}$ exon within intron 8 of the 22-intron protein tyrosine phosphatase, receptor type A (PTPRA) gene (Fig. 6c). Reminiscent of the conserved poison exons in TRA2B and U2AF2 (Fig. 1b, c), inclusion of (PTPRA) exon 8a was readily observable in the EJC libraries, but nearly undetectable in the RNA-Seq libraries (Fig. 6c). Other highabundance examples were a $103 \mathrm{nt}$ exon in intron 3 of the 29-intron DNA Polymerase 
A

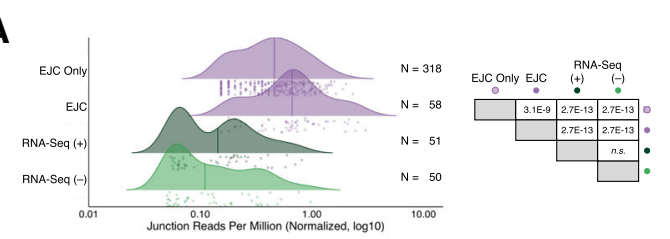

B

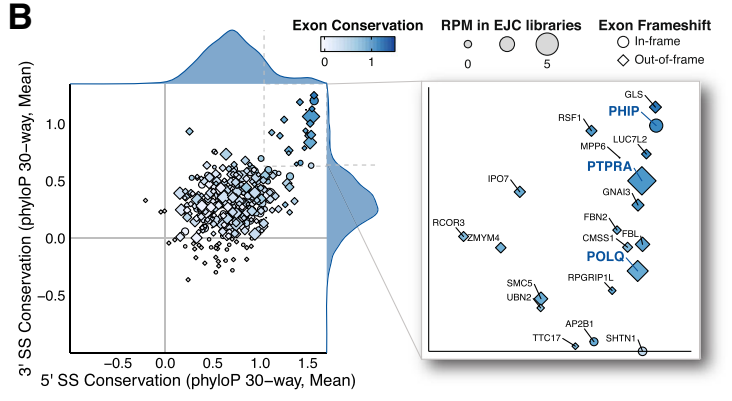

C
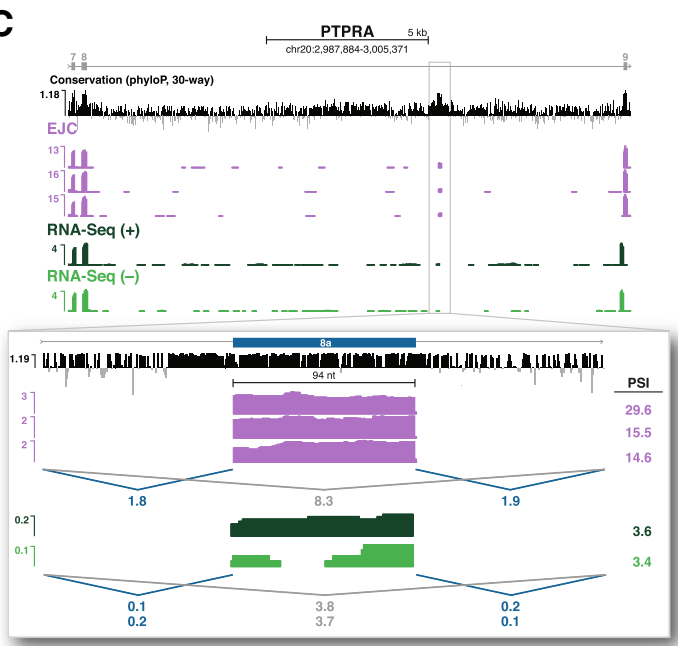

D

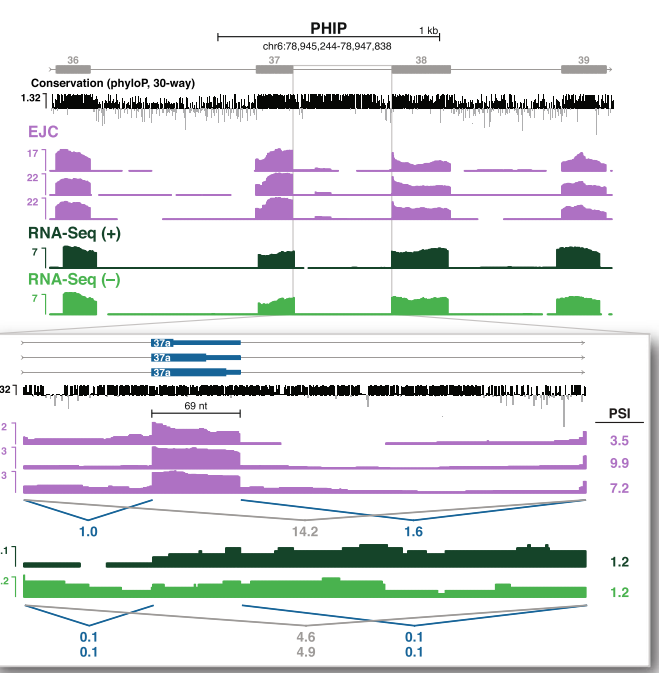

Fig. 6 (See legend on next page.) 
(See figure on previous page.)

Fig. 6 a (Left) Density plot comparing junction-spanning read coverage (RPM) for new cassette exons in EJC and RNA-Seq libraries. Line indicates median expression per library and dots represent individual cassette exons. N: number of observed cassette exons per library. (Right) Results of one-way ANOVA and Tukey's post hoc significance tests comparing the distribution of junction reads in the indicated library types. b (Left) Scatterplot comparing mean conservation (phyloP, 30-way) at 5' and 3' splice sites of new cassette exons. Exons with scores above 0 at both splice sites are colored (white to dark blue) to indicate mean exon conservation and sized by the number of junction-spanning reads supporting that exon in EJC RIPiT-Seq libraries. Diamonds indicate exons that create a frameshift in the resulting mRNA; circles indicate non-frameshifting exons. (Right) Zoomed view of exons with mean 5' and 3' splice site conservation scores above 1.04 and 0.63 , respectively. b, c Genome browser tracks of library coverage across new poison cassette exons in PHIP (b) and PTPRA (c). New cassette exons are shown in blue and numbered according to their placement in the major isoform observed in all libraries. Conservation tracks and annotations are as in Fig. 1b-d. See "Methods" for PSI formula

Theta (POLQ) gene (Additional file 1: Figure S5C) and a 69 nt exon in intron 37 of the 39-intron pleckstrin homology domain interacting protein (PHIP) gene (Fig. 6d). Although PHIP exon 37a does not frameshift, it does contain three highly conserved in-frame stop codons (Fig. 6d, bottom). Thus, all of the new evolutionarily conserved cassette exons identified here likely function as poison exons to regulate protein expression from their host gene.

\section{Discussion}

Here we demonstrate that deep sequencing of transcripts in pre-translational RNPs provides a means to identify and quantify mRNA isoforms underrepresented in or absent from RNA-Seq libraries due to their rapid elimination by translation-dependent mRNA decay. We captured this pre-translational population by tandem immunoprecipitation (RIPiT) [12] of two core EJC proteins. EJCs are stably deposited upstream of exon junctions late in the pre-mRNA splicing process, and EJCs in 5' UTRs and coding regions ( 98\% of all) are necessarily removed during the first or "pioneer" round of ribosome transit. Thus the EJC provides an excellent handle by which to enrich for fully processed, but not-yet-translated mRNAs (Fig. 1a). Our EJC RIPiT-Seq libraries enabled us to identify thousands of new exon junctions not currently annotated in any of four major reference datasets based on RNA-Seq. Many of these new splicing events generate isoforms subject to NMD, with some being evolutionarily conserved AS-NMD regulatory events. Thus EJC RIPiT-Seq constitutes a useful method to query the spliced transcriptome without the confounding effects of differential translation-dependent decay of individual mRNA isoforms.

\section{Measuring flux through AS-NMD pathways}

Since its initial description [7, 37, 38], AS-NMD has increasingly emerged as a key post-transcriptional regulatory mechanism [39-41]. Due to their widely different decay rates, however, the flux through the alternative processing pathways resulting in protein-coding and NMD isoforms cannot be captured by traditional RNA-Seq methods. As shown in Fig. 1, the vast majority of TRA2B and U2AF2 transcripts present in RNA-Seq libraries are protein-coding isoforms. The EJC RIPiT-Seq libraries, however, tell a very different story. For both TRA2B and U2AF2, the 
predominant pre-translational isoform is the poison-exon-included isoform, with poison exon PSIs averaging $88.4 \%$ and $57.5 \%$, respectively. Thus, in the cells and growth conditions examined here, alternative splicing flux for both genes strongly favors poison exon inclusion. Similar results were observed for other RNA-binding protein genes known to maintain protein homeostasis by AS-NMD (Additional file 1: Figure S1). Indeed, enrichment of transcripts subject to translation-dependent decay (e.g., isoforms annotated as NMD and NSD) is a general feature of our EJC RIPiT-Seq libraries (Fig. 2).

One can increase the abundance of transcripts subject to translation-dependent decay in RNA-Seq libraries by globally inhibiting translation prior to cell lysis [42, 43]. Indeed, to enrich for pre-translational RNPs in our RIPiT-Seq experiments, we generally expose cells to a translational inhibitor for $60 \mathrm{~min}$ prior harvest $[9,12,13,44]$. However, the harringtonine (+) and (-) RNA-Seq data in this study clearly show that this treatment had no discernible effect on either protein-coding or ASNMD isoform abundance in actively dividing HEK293 cells (Figs. 1 and 2 and Additional file 1: Figure S1-2). Another recent study provided whole cell RNA-Seq data for HeLa cells either not subjected to translation inhibition or incubated with $100 \mathrm{mg} / \mathrm{ml} \mathrm{cy-}$ cloheximide for $15 \mathrm{~min}$ or $24 \mathrm{~h}[45,46]$. While the 15-min exposure had almost no effect on protein-coding isoform abundance, the 24-h exposure did [45, 46] (Additional file 1: Figure S6A). Our analysis of those data revealed that the 15-min cycloheximide treatment was sufficient to increase AS-NMD isoform abundance, with this increase being even more apparent at $24 \mathrm{~h}$ (Additional file 1: Figure S6B). The differences we observed between HEK293 (no detectable increase in AS-NMD isoform abundance after 60 min harringtonine treatment) and HeLa (clearly detectable increase in AS-NMD isoform abundance after $15 \mathrm{~min}$ cycloheximide treatment) could be due to use of different translation inhibitors or to different mRNA synthesis and translation-dependent decay kinetics between the two cell types. Nonetheless, these data illustrate the complexities of trying to assess synthetic flux through AS-NMD pathways using translation inhibition and RNA-Seq alone.

\section{Identification of unannotated conserved splicing events}

A major goal for this study was to assess the utility of EJC RIPiT-Seq libraries for identifying sites of exon ligation underrepresented in traditional RNA-Seq libraries. As illustrated in Fig. 3a, there remain substantial differences in exon ligation events annotated in RefSeq and Ensembl/GENCODE. Further, not all exon ligation events annotated in these reference sets were returned by the CHESS pipeline, the deepest analysis of RNA-Seq to date. Here we identified thousands of exon junctions not currently annotated in RefSeq, Ensembl/GENCODE, or CHESS (Fig. 3c). Whereas the majority of these events occur at sites lacking splice site conservation (Figs. 5 and 6) and so likely constitute splicing noise, hundreds exhibit high sequence conservation among mammals. Among this conserved set, the majority display features expected to generate an AS-NMD isoform (i.e., frameshift or in-frame stop codon).

\section{New poison exons regulate genes linked to cancer}

It has now been well established that changes to pre-mRNA splicing patterns can drive both cancer initiation and cancer progression [47, 48]. Thus, it is of particular note that 
three of the most conserved, high-abundance AS-NMD events discovered here are poison cassette exons in PTPRA, PHIP, and POLQ (Fig. 6). All three genes have been linked to poor cancer prognosis when overexpressed [49-54]. While protein overexpression in cancer often results from gene duplication or transcriptional dysregulation, decreased flux through a splicing pathway leading to poison exon inclusion would have the same effect. Previous studies examining the links between NMD and cancer have focused mainly on loss of tumor suppressor genes due to increased NMD [55, 56] or the advantageous effects of NMD in eliminating mRNA isoforms encoding neoepitopes that would otherwise be recognized by the immune system [57]. But our findings suggest that decreased poison exon inclusion should also be considered as a contributor to the mechanisms underlying cancer. An obvious means to alter splicing flux is a cisacting mutation that disrupts splice site recognition and, thereby, poison exon inclusion. Although our examination of The Cancer Genome Atlas (Release 19) [58] database revealed no instances of splice site mutations associated with any of the new conserved poison cassette exons documented here, this possibility should certainly be considered in future hunts for cancer-promoting mutations. Of note, current "exome" sequencing generally captures only DNA covering and surrounding annotated exons [59]. Therefore, the unannotated cassette exons we identified here are likely absent from most DNA sequencing databases.

\section{Conclusions}

Sequencing of post-splicing, pre-translational mRNPs provides a powerful approach to identify and quantify transient species that undergo rapid translation-dependent decay and are therefore underrepresented in or completely absent from standard RNA-Seq libraries. The data here constitute just one snapshot of AS flux in HEK293 cells growing under optimal conditions. Future studies examining EJC RIPiT-Seq libraries from more diverse biological samples will undoubtedly lead to discovery of even more previously undocumented AS-NMD pathways. Examination of how flux through such pathways changes in response to changing cellular conditions will increase our general understanding of how post-transcriptional mechanisms regulate protein abundance.

\section{Methods}

Deep sequencing libraries

EJC RIPiT-Seq libraries were downloaded from the NCBI GEO GSE115788 (specifically, samples GSM3189985, GSM3189986, and GSM3189987). These libraries were generated from 200 to $550 \mathrm{nt}$ fragments by 3' adaptor ligation and reverse transcription. Paired-end sequencing (150 nt reads) on the Illumina NextSeq platform resulted in 18-24 million mate pairs per replicate [13, 14]. For RNA-Seq libraries [15], FLAG-Magoh HEK293 (validated in [9, 13]) cells were grown as in [13] and subjected (+) or not (-) to a $1 \mathrm{~h}$ treatment with harringtonine $(2 \mathrm{ng} / \mathrm{mL})$ prior to cell harvest. RNA was isolated in TRIzol using the Direct-zol RNA Kit (Zymo Research, R2062). Deep sequencing libraries were prepared from three biological replicates per condition using the KAPA RNA HyperPrep Kit with RiboErase (HMR) (Roche, 08098131702) with a modified protocol as follows. 
Isolated RNA (7 $\mu$ g; Quantified using Qubit RNA Broad Range Assay Kit (Thermo Fisher Scientific, Q10210) was first treated with Turbo DNase using the standard protocol (Thermo Fisher Scientific, AM2239). Treated RNA (1 $\mu \mathrm{g})$ was then used as input at the rRNA depletion step in the KAPA kit protocol. To generate fragments of similar size to EJC RIPiT-Seq libraries, fragmentation was carried out for $5 \mathrm{~min}$ at $94{ }^{\circ} \mathrm{C}$ and samples immediately chilled on ice. Following seven amplification cycles using the dual index adapters $(5 \mu \mathrm{L}$ of a $1.5 \mu \mathrm{M}$ per $\mu \mathrm{L}$ dilution; final concentration of $10 \mathrm{nM}$ ) from the KAPA Dual-Indexed Adapter Kit (KK8722) (Roche, 08278555702), PCR products containing 200 to $550 \mathrm{nt}$ inserts were size selected using SPRIselect beads (Beckman Coulter Life Sciences, B23318) and quantified using the Qubit dsDNA Broad Range Assay Kit (Thermo Fisher Scientific, Q32850). As each library contained a unique barcode, all libraries (3 biological replicates + or - harringtonine treatment) were mixed and sequenced on an Illumina NextSeq 550 instrument using the High Output Kit v2.5 (Illumina, Inc., 20,024,908). All libraries were loaded at $1.8 \mathrm{pm}$ with 5\% PhiX and all data was written to BaseSpace (Illumina, Inc.).

\section{Library processing and alignment}

Read counts for unprocessed libraries and for the individual processing steps detailed below are provided in Additional file 1: Table S1. Prior to alignment, adaptor sequences and long stretches $(\geq 20 \mathrm{nt}$ ) of adenosines were trimmed from read 3' ends. All libraries were filtered for reads aligning to repeat regions, as defined by RepeatMasker [18], using STAR v2.5.3a [17]. Remaining reads were aligned with STAR on two-pass mode to the human genome, release 93 [3]. This alignment allowed a maximum of 3 mismatches per pair and highly penalized deletions and insertions. Mapped reads were then filtered for low mapping quality (MAPQ <5) and/or duplicated reads, identified with the MarkDuplicates tool (Picard v2.17.8) [60].

\section{RNA isoform quantification}

RNA isoform abundances were determined using Kallisto (v0.44.0) [61], using only reads that passed the filtering and alignment steps described above. Transcript biotypes (i.e., "protein-coding," "nonsense-mediated decay") and intron counts used to categorize transcripts throughout Fig. 2, Additional file 1: Figure S2 and S6A are based on the transcriptome annotation from Ensembl (GRCh38.p12) [3].

\section{Junction identification pipeline}

The custom bioinformatics pipeline designed for our annotated and unannotated junction analysis (Figs. 3, 4, 5, and 6) is shown in detail in Additional file 1: Figure S3A. Transcriptome annotation files from RefSeq (hg38) [23], Ensembl (GRCh38.p12) [3], GENCODE (v29) [24], and CHESS (v2.1) [26] were combined to create a comprehensive reference file of all annotated introns (Additional file 2: Table S2). Any junction that appears in our libraries but is not annotated in one of the aforementioned transcriptomes is referred to as "unannotated." 
To identify unannotated exon junctions, all reads with CIGAR strings containing an "N" operation were isolated and compared to the annotated intron reference file using Bedtools intersect [62]. Reads with alignment gaps not matching the length or location of a known intron were considered the result of potential unannotated splicing events. These junctions were further filtered based on the following criteria: (i) overlap with a known gene; (ii) reads must have $\geq 15 \mathrm{nt}$ aligned on both sides of the potential junction; (iii) present in all replicates of any library type; (iv) GT and AG dinucleotides at the $5^{\prime}$ and $3^{\prime}$ splice sites, respectively; and (v) mean read count $\geq 2$ per library type.

\section{Nearest annotated splice site analysis}

For analysis of new splicing events near annotated exons (Fig. 4), each unannotated 5' splice site was paired with its nearest annotated 5' splice site based on the $3^{\prime}$ splice site used in both splicing events. Similarly, each unannotated 3' splice site was paired with its nearest annotated 3' splice site based on the $5^{\prime}$ splice site used in both splicing events. The number of available GT and AG dinucleotides at nucleotide positions - 30 to +30 surrounding each annotated splice site in this unannotated/annotated paired dataset was counted to determine the frequency of potential splice sites in the relevant region.

\section{Splice site strength and conservation}

Splice site strength and mean conservation scores for annotated and unannotated splice sites were calculated using MaxEntScan [36] and phyloP 30-way basewise conservation scores [35] (Fig. 5a). Random sequences of the appropriate length ( 9 nts for 5 ' splice sites and 23 nts for $3^{\prime}$ splice sites) and internal to annotated genes were obtained from the hg38 annotation file [3] using the Bedtools random function [62]. Only those random sequences containing a GT at positions 4 and 5 or an AG at positions 19 and 20 were used to calculate MaxENT and conservation scores for comparison to $5^{\prime}$ and $3^{\prime}$ splice sites, respectively.

\section{Plotting and data visualization}

Data visualization was performed in R [63] using ggplot2 [64], ggrepel [65], UpSetR [27], ggseqlogo [28], eulerr [29], and ggridges [66] software packages. The UCSC Genome Browser $[16,67]$ was used to view library tracks and to create transcript figures throughout the manuscript.

\section{Splice site usage calculations}

The following equations were used to calculate RPM, PSI, PSO, and PIR values throughout the manuscript. NMD and PC refer to Nonsense-Mediated Decay and Protein Coding isoforms, respectively. 


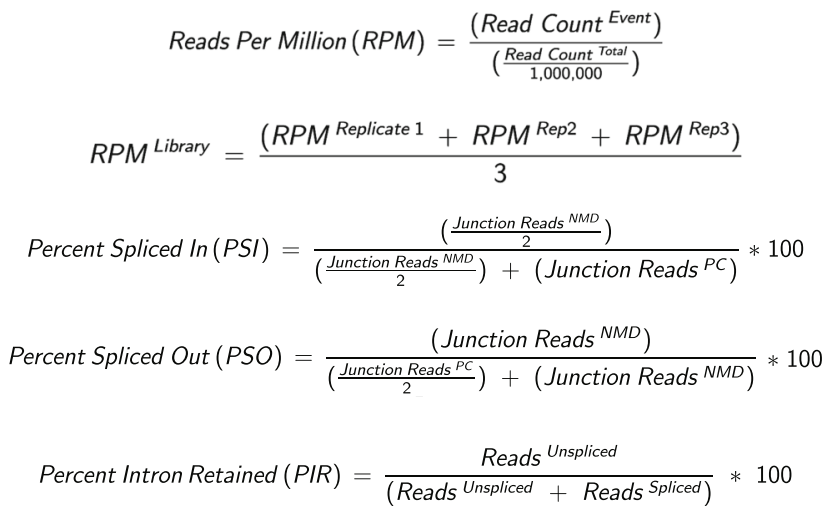

\section{Supplementary Information}

Supplementary information accompanies this paper at https://doi.org/10.1186/s13059-021-02309-y.

Additional file 1: Figure S1-S5 and Table S1. Figure S1. Comparison of biological replicates; additional examples of AS-NMD transcript coverage. Figure S2. Comparison of EJC and RNA-Seq libraries coverage across additional transcript biotypes. Figure $\mathbf{S 3}$. Representation of bioinformatics pipeline to classify annotated and unannotated exon junctions. Figure S4. Comparison of MaxENT and conservation scores for annotated and randomly located splice sites; additional examples of conserved unannotated splicing events. Figure S5. Additional example of an unannotated AS-NMD cassette exon. Figure S6. Analysis of previously published RNA-Seq libraries made from HeLa cells treated with cycloheximide for $0 \mathrm{~min}, 15 \mathrm{~min}$, or $24 \mathrm{~h}[45,46]$. Table S1. Sequencing and alignment information for each replicate of the analyzed libraries.

Additional file 2: Table S2. Comprehensive file of all intron locations annotated in RefSeq (hg38), Ensembl (GRCh38.p12), GENCODE (v29), and CHESS (v2.1).

Additional file 3: Table S3. Locations and characteristics of highly conserved unannotated exon junctions identified in this study.

Additional file 4. Includes peer review history.

\section{Acknowledgements}

We thank Athma Pai, Harleen Saini, and Guramrit Singh for critical reading of the manuscript. We thank Weijun Chen for technical advice. We thank Wei Zheng and Chiao-wen Hsiao for advice on statistical analysis.

\section{Author details}

M.J.M. is a member of the National Academy of Science (USA) and a fellow of the American Association of Arts and Sciences.

\section{Peer review information}

Andrew Cosgrove was the primary editor on this article and managed its editorial process and peer review in collaboration with the rest of the editorial team.

Review history

The review history is available as Additional file 4.

\section{Authors' contributions}

M.J.M conceived of the project. C.K. and M.J.M were responsible for all data analysis and manuscript writing. S.D. and A.A.B. constructed the RNA-Seq libraries and participated in manuscript editing. M.M. provided technical advice regarding the EJC RIPiT-Seq libraries and participated in manuscript editing. The author(s) read and approved the final manuscript.

\section{Authors' information}

Twitter handles: @MihirMetkar (Mihir Metkar); @mooreorless62 (Melissa J. Moore).

\section{Funding}

This work was supported by funding from HHMI and NIH RO1-GM53007. M.J.M. was an HHMI Investigator during the time this work was conducted.

\section{Availability of data and materials}

The RIPiT-Seq datasets analyzed in this study were downloaded from NCBI GEO under accession number GSE115788 (specifically, samples GSM3189985, GSM3189986, and GSM3189987) [14]. The whole cell RNA-Seq data from HeLa cells either not subjected to translation inhibition or treated with cycloheximide were downloaded from NCBI GEO under accession number GSE125086 (specifically, samples GSM3562588, GSM3562589, GSM3562590, GSM3562591, 
GSM3562592, GSM3562593) [46]. The accession number for the RNA-Seq raw fastq files reported in this paper is GSE150228 [15].

\section{Declarations}

\section{Ethics approval and consent to participate}

Not applicable.

\section{Consent for publication}

Not applicable.

\section{Competing interests}

S.D., A.A.B., M.M., and M.J.M. are currently employees of and shareholders in Moderna. M.J.M. is also a shareholder and scientific advisory board member for Arrakis Therapeutics.

\section{Author details}

${ }^{1}$ RNA Therapeutics Institute, University of Massachusetts Medical School, Worcester, MA 01605, USA. ²Department of Biochemistry and Molecular Pharmacology, University of Massachusetts Medical School, Worcester, MA 01605, USA.

${ }^{3}$ Present Address: Moderna, 200 Technology Square, Cambridge, MA 02139, USA.

Received: 7 October 2019 Accepted: 2 March 2021

Published online: 03 May 2021

\section{References}

1. Wang ET, Sandberg R, Luo S, Khrebtukova I, Zhang L, Mayr C, et al. Alternative isoform regulation in human tissue transcriptomes. Nature. 2008. https://doi.org/10.1038/nature07509.

2. Pan $\mathrm{Q}$, Shai $\mathrm{O}$, Lee $L J$, Frey BJ, Blencowe BJ. Deep surveying of alternative splicing complexity in the human transcriptome by high-throughput sequencing. Nat Genet. 2008. https://doi.org/10.1038/ng.259.

3. Cunningham F, Achuthan P, Akanni W, Allen J, Amode MR, Armean IM, et al. Ensembl 2019. Nucleic Acids Res. 2019. https://doi.org/10.1093/nar/gky1113.

4. Nilsen TW, Graveley BR. Expansion of the eukaryotic proteome by alternative splicing. Nature. 2010. https://doi.org/10.1 038/nature08909.

5. Kelemen O, Convertini P, Zhang Z, Wen Y, Shen M, Falaleeva M, et al. Function of alternative splicing. Gene. 2013 https://doi.org/10.1016/j.gene.2012.07.083.

6. Kurosaki T, Popp MW, Maquat LE. Quality and quantity control of gene expression by nonsense-mediated mRNA decay. Nat Rev Mol Cell Biol. 2019. https://doi.org/10.1038/s41580-019-0126-2.

7. Lewis BP, Green RE, Brenner SE. Evidence for the widespread coupling of alternative splicing and nonsense-mediated mRNA decay in humans. PNAS. 2003. https://doi.org/10.1073/pnas.0136770100.

8. Nasif S, Contu L, Mühlemann O. Beyond quality control: the role of nonsense-mediated mRNA decay (NMD) in regulating gene expression. Semin Cell Dev Biol. 2018. https://doi.org/10.1016/j.semcdb.2017.08.053.

9. Singh G, Kucukural A, Cenik C, Leszyk JD, Shaffer SA, Weng Z, et al. The cellular EJC interactome reveals higher-order mRNP structure and an EJC-SR protein nexus. Cell. 2012. https://doi.org/10.1016/j.cell.2012.10.007.

10. Saulière J, Murigneux V, Wang Z, Marquenet E, Barbosa I, Le Tonquèze O, et al. CLIP-seq of elF4All reveals transcriptome-wide mapping of the human exon junction complex. NSMB. 2012. https://doi.org/10.1038/nsmb.2420

11. Maquat $L E$, Tarn WY, Isken O. The pioneer round of translation: features and functions. Cell. 2010. https://doi.org/10.101 6/j.cell.2010.07.022.

12. Singh G, Ricci EP, Moore MJ. RIPiT-Seq: a high-throughput approach for footprinting RNA:protein complexes. Methods. 2014. https://doi.org/10.1016/j.ymeth.2013.09.013.

13. Metkar M, Ozadam H, Lajoie BR, Imakaev M, Mirny LA, Dekker J, et al. Higher-order organization principles of pretranslational mRNPs. Mol Cell. 2018. https://doi.org/10.1016/.molcel.2018.09.012.

14. Metkar M, Ozadam H, Lajoie BR, Imakaev M, Mirny LA, Dekker J, et al. Higher-order organization principles of pretranslational mRNPs. Datasets. Gene Expression Omnibus. 2018; https:/www.ncbi.nlm.nih.gov/geo/query/acc.cgi?acc= GSE115788. Accessed 19 Feb 2021.

15. Kovalak C, Donovan S, Bicknell AA, Metkar M, Moore MJ. Deep sequencing of pre-translational mRNPs reveals hidden flux through evolutionarily conserved AS-NMD pathways. Datasets. Gene Expression Omnibus. 2021; https://www.ncbi. nlm.nih.gov/geo/query/acc.cgi?acc=GSE150228. Accessed 19 Feb 2021

16. Kent WJ, Sugnet CW, Furey TS, Roskin KM, Pringle TH, Zahler AM, et al. The human genome browser at UCSC. Genome Res. 2002. https://doi.org/10.1101/gr.229102.

17. Dobin A, Davis CA, Schlesinger F, Drenkow J, Zaleski C, Jha S, et al. STAR: ultrafast universal RNA-seq aligner. Bioinformatics. 2013. https://doi.org/10.1093/bioinformatics/bts635.

18. Smit AFA, Hubley R, Green, P. RepeatMasker Open-4.0. 2013-2015. http://www.repeatmasker.org. Accessed 04 Sept 2019

19. http://vega.archive.ensembl.org/info/about/gene_and_transcript_types.html. Accessed 04 Sept 2019.

20. Klauer AA, van Hoof A. Degradation of mRNAs that lack a stop codon: a decade of nonstop progress. Wiley Interdiscip Rev RNA. 2012. https://doi.org/10.1002/wrna.1124.

21. Van den Berge K, Hembach KM, Soneson C, Tiberi S, Clement L, Love MI, et al. RNA sequencing data: Hitchhiker's guide to expression analysis. Annu Rev Biomed Data Sci. 2019. https://doi.org/10.1146/annurev-biodatasci-072018-021255.

22. Soneson C, Matthes KL, Nowicka M, Law CL, Robinson MD. Isoform prefiltering improves performance of count-based methods for analysis of differential transcript usage. Genome Biol. 2016. https://doi.org/10.1186/s13059-015-0862-3.

23. O'Leary NA, Wright MW, Brister JR, Ciufo S, Haddad D, McVeigh R, et al. Reference sequence (RefSeq) database at NCBI: current status, taxonomic expansion, and functional annotation. Nucleic Acids Res. 2016. https://doi.org/10.1093/nar/ gkv1189. 
24. Frankish A, Diekhans M, Ferreira A, Johnson R, Jungreis I, Loveland J, et al. GENCODE reference annotation for the human and mouse genomes. Nucleic Acids Res. 2019. https://doi.org/10.1093/nar/gky955.

25. GENCODE. https://www.gencodegenes.org/pages/faq.html. Accessed 02 Apr 2020.

26. Pertea M, Shumate A, Pertea G, Varabyou A, Breitwieser FP, Chang YC, et al. CHESS: a new human gene catalog curated from thousands of large-scale RNA sequencing experiments reveals extensive transcriptional noise. Genome Biol. 2018. https://doi.org/10.1186/s13059-018-1590-2.

27. Conway JR, Lex A, Gehlenborg N. UpSetR: an R package for the visualization of intersecting sets and their properties Bioinformatics. 2017. https://doi.org/10.1093/bioinformatics/btx364.

28. Wagih O. ggseqlogo: a versatile R package for drawing sequence logos. Bioinformatics. 2017. https://doi.org/10.1093/ bioinformatics/btx469.

29. Larsson J. eulerr: area-proportional Euler and Venn diagrams with ellipses. R package version 5.1.0. 2019. https://cran.rproject.org/package=eulerr. Accessed 04 Sept 2019.

30. Dou Y, Fox-Walsh KL, Baldi PF, Hertel KJ. Genomic splice-site analysis reveals frequent alternative splicing close to the dominant splice site. RNA. 2006. https://doi.org/10.1261/rna.151106.

31. Pickrell JK, Pai AA, Gilad Y, Pritchard JK. Noisy splicing drives mRNA isoform diversity in human cells. PLoS Genet. 2010 https://doi.org/10.1371/journal.pgen.1001236.

32. Miriami E, Motro U, Sperling J, Sperling R. Conservation of an open-reading frame as an element affecting $5^{\prime}$ splice site selection. J Struct Biol. 2002. https://doi.org/10.1016/S1047-8477(02)00539-7.

33. Wachtel C, Li B, Sperling J, Sperling R. Stop codon-mediated suppression of splicing is a novel nuclear scanning mechanism not affected by elements of protein synthesis and NMD. RNA. 2004. https://doi.org/10.1261/rna.7480804.

34. Fox-Walsh KL, Hertel K. Splice-site pairing is an intrinsically high fidelity process. PNAS. 2009. https://doi.org/10.1073/ pnas.0813128106.

35. Pollard KS, Hubisz MJ, Rosenbloom KR, Siepel A. Detection of nonneutral substitution rates on mammalian phylogenies. Genome Res. 2010. https://doi.org/10.1101/gr.097857.109.

36. Yeo G, Burge CB. Maximum entropy modeling of short sequence motifs with applications to RNA splicing signals. J Comput Biol. 2004 https://doi.org/10.1089/1066527041410418

37. Morrison M, Harris KS, Roth MB. smg mutants affect the expression of alternatively spliced SR protein mRNAs in Caenorhabditis elegans. PNAS. 1997. https://doi.org/10.1073/pnas.94.18.9782.

38. Mitrovich QM, Anderson P. Unproductively spliced ribosomal protein mRNAs are natural targets of mRNA surveillance in C. elegans. Genes Dev. 2000. https://doi.org/10.1101/gad.819900.

39. Zheng S, Gray EE, Chawla G, Porse BT, O'Dell TJ, Black DL. PSD-95 is post-transcriptionally repressed during early neural development by PTBP1 and PTBP2. Nat Neurosci. 2012. https://doi.org/10.1038/nn.3026.

40. Hamid FM, Makeyev EV. Regulation of mRNA abundance by polypyrimidine tract-binding protein-controlled alternate $5^{\prime}$ splice site choice. PLoS Genet. 2014. https://doi.org/10.1371/journal.pgen.1004771

41. Yan Q, Weyn-Vanhentenryck SM, Wu J, Sloan SA, Zhang Y, Chen K, et al. Systematic discovery of regulated and conserved alternative exons in the mammalian brain reveals NMD modulating chromatin regulators. PNAS. 2015. https://doi.org/10.1073/pnas.1502849112

42. Carter MS, Doskow J, Morris P, Li S, Nhim RP, Sandstedt S, et al. A regulatory mechanism that detects premature nonsense codons in T-cell receptor transcripts. Biol Chem. 1995. https://doi.org/10.1074/jbc.270.48.28995.

43. Sidhu JS, Omiecinski CJ. Protein synthesis inhibitors exhibit a nonspecific effect on phenobarbital-inducible cytochome P450 gene expression in primary rat hepatocytes. J Comp Biol. 1998. https://doi.org/10.1074/jbc.273.8.4769.

44. Mabin JW, Woodward LA, Patton RD, Yi Z, Jia M, Wysocki VH, et al. The exon junction complex undergoes a compositional switch that alters mRNP structure and nonsense-mediated mRNA decay activity. Cell Rep. 2018. https:// doi.org/10.1016/j.celrep.2018.11.046.

45. Kearse MG, Goldman DH, Choi J, Nwaezeapu C, Liang D, Green KM, et al. Ribosome queuing enables non-AUG translation to be resistant to multiple protein synthesis inhibitors. Genes Dev. 2019. https://doi.org/10.1101/gad.324715.119.

46. Kearse MG, Goldman DH, Choi J, Nwaezeapu C, Liang D, Green KM, et al. Ribosome queuing enables non-AUG translation to be resistant to multiple protein synthesis inhibitors. Datasets. Gene Expression Omnibus. 2019; https:// www.ncbi.nIm.nih.gov/geo/query/acc.cgi?acc=GSE125086. Accessed 19 Feb 2021.

47. Sveen A, Kilpinen S, Ruusulehto A, Lothe RA, Skotheim Rl. Aberrant RNA splicing in cancer; expression changes and driver mutations of splicing factor genes. Oncogene. 2016. https://doi.org/10.1038/onc.2015.318.

48. Climente-González H, Porta-Pardo E, Godzik A, Eyras E. The functional impact of alternative splicing in cancer. Cell Rep. 2017. https://doi.org/10.1016/i.celrep.2017.08.012

49. Tabiti K, Smith DR, Goh HS, Pallen CJ. Increased mRNA expression of the receptor-like protein tyrosine phosphatase alpha in late stage colon carcinomas. Cancer Lett. 1995. https://doi.org/10.1016/0304-3835(95)03816-f.

50. Ardini E, Agresti R, Tagliabue E, Greco M, Aiello P, Yang LT, et al. Expression of protein tyrosine phosphatase alpha (RPTPa) in human breast cancer correlates with low tumor grade, and inhibits tumor cell growth in vitro and in vivo. Oncogene. 2000. https://doi.org/10.1038/sj.onc.1203869.

51. Gu Z, Fang X, Li C, Chen C, Liang G, Zheng X, et al. Increased PTPRA expression leads to poor prognosis through c-Src activation and G1 phase progression in squamous cell lung cancer. Int J Oncol. 2017. https://doi.org/10.3892/ijo.2017.4055.

52. De Semir D, Nosrati M, Bezrookove V, Dar AA, Federman S, Bienvenu G, et al. Pleckstrin homology domain-interacting protein (PHIP) as a marker and mediator of melanoma metastasis. PNAS. 2012. https://doi.org/10.1073/pnas.1119949109.

53. Wood RD, Doublié S. DNA polymerase $\theta$ (POLQ), double-strand break repair, and cancer. DNA Repair. 2016. https://doi. org/10.1016/j.dnarep.2016.05.003.

54. Goullet de Rugy T, Bashkurov M, Datti A, Betous R, Guitton-Sert L, Cazaux C, et al. Excess Pol $\theta$ functions in response to replicative stress in homologous recombination-proficient cancer cells. Biol Open. 2016. https:/doi.org/10.1242/bio.018028.

55. Lindeboom RG, Supek F, Lehner B. The rules and impact of nonsense-mediated mRNA decay in human cancers. Nat Genet. 2016. https://doi.org/10.1038/ng.3664.

56. Hu Z, Yau C, Ahmed AA. A pan-cancer genome-wide analysis reveals tumour dependencies by induction of nonsensemediated decay. Nat Commun. 2017. https://doi.org/10.1038/ncomms15943. 
57. Pastor F, Kolonias D, Giangrande PH, Gilboa E. Induction of tumour immunity by targeted inhibition of nonsensemediated mRNA decay. Nature. 2010. https://doi.org/10.1038/nature08999.

58. Cancer Genome Atlas Research Network, Weinstein JN, Collisson EA, Mills GB, Shaw KR, Ozenberger BA, et al. The cancer genome atlas pan-cancer analysis project. Nat Genet. 2013. https://doi.org/10.1038/ng.2764.

59. Wang VG, Kim H, Chuang JH. Whole-exome sequencing capture kit biases yield false negative mutation calls in TCGA cohorts. PLoS One. 2018. https://doi.org/10.1371/journal.pone.0204912.

60. Picard. http://broadinstitute.github.io/picard. Accessed 04 Sept 2019

61. Bray NL, Pimentel H, Melsted P, Pachter L. Near-optimal probabilistic RNA-seq quantification. Nat Biotechnol. 2016. https://doi.org/10.1038/nbt.3519.

62. Quinlan AR, Hall IM. BEDTools: a flexible suite of utilities for comparing genomic features. Bioinformatics. 2010 https://doi.org/10.1093/bioinformatics/btq033.

63. R Core Team. R: a language and environment for statistical computing. R Foundation for statistical computing. Vienna; 2016. http://www.R-project.org/. Accessed on 04 Sept 2019

64. Wickham H. ggplot2: elegant graphics for data analysis. New York: Springer-Verlag; 2016

65. Slowikowski K. ggrepel: automatically position non-overlapping text labels with 'ggplot2'. R package version 0.8.0. 2018. https://CRAN.R-project.org/package=ggrepel. Accessed on 04 Sept 2019.

66. Wilke CO. ggridges: ridgeline plots in 'ggplot2'. R package version 0.5.1. 2018. https://CRAN.R-project.org/package= ggridges. Accessed on 04 Sept 2019.

67. Raney BJ, Dreszer TR, Barber GP, Clawson H, Fujita PA, Wang T, et al. Track data hubs enable visualization of userdefined genome-wide annotations on the UCSC genome browser. Bioinformatics. 2014. https://doi.org/10.1093/ bioinformatics/btt637.

\section{Publisher's Note}

Springer Nature remains neutral with regard to jurisdictional claims in published maps and institutional affiliations.

Ready to submit your research? Choose BMC and benefit from:

- fast, convenient online submission

- thorough peer review by experienced researchers in your field

- rapid publication on acceptance

- support for research data, including large and complex data types

- gold Open Access which fosters wider collaboration and increased citations

- maximum visibility for your research: over $100 \mathrm{M}$ website views per year

At $B M C$, research is always in progress.

Learn more biomedcentral.com/submissions 